\title{
Anemia e marcadores séricos da deficiência de ferro em grávidas atendidas na rede pública municipal de Manaus, Amazonas, Brasil
}

\begin{abstract}
Carolina Marinho da COSTA ${ }^{1}$, Ione Rodrigues BRUM², Emerson Silva LIMA ${ }^{3}$
RESUMO

O presente estudo teve como objetivo avaliar a anemia em grávidas, associando os resultados da dosagem de hemoglobina e hematócrito com a análise de marcadores do perfil sérico do ferro. Participaram do estudo 92 grávidas que estavam realizando pré-natal em unidades de atendimento à saúde no Município de Manaus, Amazonas, Brasil. Foi aplicado um formulário para obtenção dos dados antropométricos e informaçóes sobre estilo de vida, além de serem realizadas dosagens dos níveis séricos de ferro, capacidade latente de ligaçáo do ferro (CLLF), capacidade total de ligação do ferro (CTLF), índice de saturação da transferrina (IST), transferrina, ferritina e níveis sanguíneos de hemoglobina e hematócrito por metodologia automatizada utilizando kits comerciais disponíveis. Foram encontradas $26,1 \%$ de grávidas com níveis de hemoglobina abaixo de $11 \mathrm{~g} / \mathrm{dL}$. Observou-se que 17,4\% das grávidas com níveis normais de hemoglobina apresentavam níveis inadequados de ferro sérico e 9,8\% apresentavam níveis baixos de ferritina sérica. Os níveis de ferritina e de hemoglobina apresentaram diferença significativa entre os trimestres de gestação ( $\mathrm{p}<0,05$, ANOVA). Os resultados sugerem que a dosagem da hemoglobina juntamente com outros marcadores do perfil sérico do ferro pode trazer uma avaliação mais precisa da deficiência de ferro na gravidez.
\end{abstract}

PALAVRAS-ChaVE: Gravidez, Ferro, Anemia, Hemoglobina.

\section{Anemia and serum markers of iron deficiency in pregnant women attended by Public Health Service in Manaus, Amazonas, Brazil}

\begin{abstract}
This study aimed to evaluate anemia in pregnant women, comparing results of hemoglobin and hemotocrit dosages with analysis of iron serum status markers. 92 pregnant women, in pre-natal attendance in Public Health Service of Manaus, Amazonas, Brazil, participated in this study. A=questionnaire was used to access anthropometric data and life style information. Serum levels of iron, latent iron binding capacity (LIBC), total iron binding capacity (TIBC), transferrin saturation (TS), transferrin, ferritin, and blood levels of hemoglobin and hematocrit were measured by automated methods, using commercially available kits. The study showed that $26.1 \%$ of the pregnant women had hemoglobin levels below $11 \mathrm{~g} / \mathrm{dL}$. Also, of the pregnant women with normal levels of hemoglobin, $17.4 \%$ showed inadequate iron levels and $9.8 \%$ low levels of ferritin. Ferritin and hemoglobin levels showed a significant statistical difference=among the trimester pregnant women $(\mathrm{p}<0.05$, ANOVA). Theses results suggest that the dosage of hemoglobin together with other iron serum markers can make for a more specific evaluation of iron deficiency in pregnancy.
\end{abstract}

KEYWORDS: Pregnancy, Iron, Anemia, Hemoglobin.

1 Universidade Federal do Amazonas - UFAM. E-mail: costacarolmarinho@hotmail.com

2 Universidade Federal do Amazonas - UFAM. E-mail: ionehelder@uol.com.br

${ }^{3}$ Universidade Federal do Amazonas - UFAM. E-mail: eslima@ufam.edu.br 


\section{INTRODUÇÃO}

A deficiência de ferro é o agravo nutricional mais prevalente no mundo, afetando principalmente lactentes, préescolares, adolescentes e grávidas. A principal conseqüência dessa deficiência é a anemia, que pode ser definida como uma redução anormal da concentração de hemoglobina no sangue em níveis menores que $11 \mathrm{~g} / \mathrm{dL}$ (Paiva et al., 2000). A anemia também é uma das principais complicaçóes da gravidez. Dependendo da gravidade, pode levar à morte não somente por problema cardiovascular como também através de hemorragia e sepse (WHO, 2000).

Estima-se que, de cada dez gestantes que fazem o prénatal, três são anêmicas (Brasil, 2003). A deficiência de ferro, na sua forma mais grave, resulta no que se denomina anemia ferropênica. Uma vez que a concentração de hemoglobina é um parâmetro fácil de se determinar, a prevalência de anemia vem sendo usada como um substituto da anemia ferropênica. Apesar dessa abordagem ser útil em lugares onde o principal tipo de anemia é o por deficiência de ferro, o mesmo não é válido em lugares onde a etiologia da anemia é mais complexa e também para detectar casos mais precoces da deficiência (WHO, 2004).

O aumento do volume circulatório materno é uma das mudanças fisiológicas mais marcantes durante a gravidez, o qual pode alcançar até $50 \%$ do volume pré-gestacional na trigésima semana de gestação (Souza et al., 2002). Tal condiçáo dificulta o diagnóstico correto da anemia, uma vez que a concentração da hemoglobina é alterada pela hemodiluição em diversos graus. Sendo assim, seria ideal conhecer o status de ferro da mulher no período pré-concepcional, a fim de se verificar se a mesma iniciou ou não a gravidez com estoque suficiente desse mineral (Souza \& Batista Filho, 2003).

Em regióes onde existem recursos adequados de diagnóstico, a hemoglobina deveria ser interpretada sempre que possível em conjunto com outros critérios para se fornecer um diagnóstico mais seguro, uma vez que a utilização de mais de um marcador aumenta consideravelmente a especificidade do diagnóstico. Entretanto, em regiōes que apresentam recursos de saúde precários e onde a anemia é muito prevalente, a hemoglobina e o hematócrito podem ser utilizados como testes de triagem ou até mesmo como diagnóstico final, visto que a concentração de hemoglobina reflete uma situação de deficiência nutricional já estabelecida (Souza \& Batista Filho, 2003).

Os parâmetros mais específicos para a determinaçáo do status do ferro são: ferro sérico, ferritina sérica, capacidade de ligação total do ferro, índice de saturação da transferrina, protoporfirina eritrocitária e, mais recentemente, o receptor de transferrina. Nos casos em que o paciente já está sob terapêutica de ingestáo de ferro, deve-se fazer a análise do ferro de depósito (ferritina), que poderá dar informaçâo mais exata sobre a falta de ferro (Verrastro, 2002). Atualmente, a dosagem da ferritina sérica, na ausência de infecção ou inflamação, é o parâmetro mais precoce para a identificaçáo da depleção dos estoques de ferro (Papa et al., 2003). É uma medida útil, pois utiliza sangue periférico e apresenta forte correlaçáo com o ferro em depósito nos tecidos (Paiva et al., 2000).

O presente trabalho teve como objetivo avaliar o status do ferro em grávidas atendidas na rede pública de saúde do município de Manaus, comparando os resultados obtidos através da dosagem de hemoglobina com parâmetros de análise do perfil sérico do ferro no diagnóstico da anemia na gravidez.

\section{MATERIAL E MÉTODOS}

\section{CASUÍSTICA}

Trata-se de um estudo descritivo transversal realizado no período de agosto a outubro de 2006. A população deste estudo foi constituída de 92 gestantes em atendimento pré-natal em Postos de Saúde da rede pública municipal de Manaus/ Amazonas/Brasil. Dados sobre condiçóes socioeconômicas, história familiar de doenças, histórico obstétrico-ginecológico e dados antropométricos (peso pré-gestacional e altura) foram obtidos através de entrevista. Para coleta desses dados utilizouse um formulário padronizado aplicado à gestante. $\mathrm{O}$ estudo foi aprovado pelo Comitê de Ética em Pesquisa com Seres Humanos da Universidade Federal do Amazonas.

\section{AMOSTRA BIOLÓGICA}

Amostras de sangue $(10 \mathrm{~mL})$ foram colhidas à vácuo em tubos secos (sem anticoagulante) e tubos contendo EDTA para realizaçâo da dosagem de hemoglobina. O tubo seco foi em seguida centrifugado a $2500 \mathrm{rpm}$ por $10 \mathrm{~min}$ para a obtenção do soro utilizado nas análises bioquímicas.

\section{ANÁLISES LABORATORIAIS}

As dosagens do hematócrito e da hemoglobina foram realizadas de forma automatizada utilizando o equipamento KX-21N (Sysmex). As dosagens de ferro sérico, capacidade latente de ligação do ferro e ferritina foram realizadas imediatamente após a coleta por método colorimétrico ou turbidimétrico utilizando o equipamento COBAS MIRA PLUS ${ }^{\circledR}$ (Roche Corp.) e kits comerciais da Labtest Diagnóstica (Minas Gerais, Brasil). Os valores de capacidade total de ligação do ferro, índice de saturação da transferrina e transferrina foram obtidos através de cálculos baseados nos níveis de ferro e capacidade latente de ligação do ferro. Foram considerados valores normais para o ferro sérico dosagem entre 50 e $150 \mu \mathrm{g} / \mathrm{dl}$, para capacidade latente de ligação do ferro entre 140 e $280 \mu \mathrm{g} / \mathrm{dL}$, para a capacidade total de ligação do ferro entre 250 a $410 \mu \mathrm{g} / \mathrm{dL}$, para o índice de saturação da transferrina entre 20 a 50\%, 
para a transferrina, entre 200 e $300 \mathrm{mg} / \mathrm{dl}$ e para a ferritina entre 10 e $120 \mathrm{ng} / \mathrm{mL}$, para hemoglobina, igual ou acima de $11 \mathrm{~g} / \mathrm{dL}$ e para o hematócrito, valores iguais ou acima de 33\% (Paiva et al., 2000).

\section{ANÁLISE ESTATÍSTICA}

Os resultados foram expressos em percentagem e como média e desvio padrão. A hipótese de igualdade de duas variáveis foi verificada fazendo uso do teste $t$ de Student e para comparação de mais de mais de duas medias foi utilizado uma análise de variância (ANOVA). Para os testes de associação foi utilizado o Teste do Qui-quadrado. O nível de significância considerado para os testes foi de $\mathrm{p}<0,05$.

\section{RESULTADOS E DISCUSSÃO}

Foram realizadas dosagens sanguíneas do hemograma e marcadores séricos da deficiência de ferro em 92 grávidas, que apresentavam em média $23 \pm 6$ anos de idade e encontravamse com $19 \pm 9$ semanas de gestação. Quanto ao índice de massa corpórea (IMC), 48\% iniciaram a gestação com o índice adequado, de acordo com os critérios adotados pela Associação Brasileira para o Estudo da Obesidade e da Síndrome Metabólica (ABESO, 2007). A prevalência de anemia foi de $26,1 \%$, considerando a concentraçáo de hemoglobina inferior a $11 \mathrm{~g} / \mathrm{dL}$, conforme a recomendação da WHO (2004). Os níveis do hematócrito e do ferro sérico foram menores naquelas consideradas anêmicas (Tabela 1). Os demais índices analisados como a capacidade latente de ligação do ferro (CLLF), capacidade total de ligaçáo do ferro (CTLF), índice de saturação da transferrina (IST), transferrina e ferritina séricas não foram diferentes entres gestantes com e sem anemia (Tabela 1).

No presente estudo não foi observada associação significativa entre os níveis de ferro sérico e hemoglobina, mesmo havendo uma proporçáo de $17,4 \%$ das gestantes com hemoglobina maior que $11 \mathrm{~g} / \mathrm{dL}$, entre aquelas $(56,5 \%) \mathrm{com}$ os níveis de ferro sérico normal ( $>50 \mu \mathrm{g} / \mathrm{dL}$ ) (Tabela 2). Além disso, das grávidas com níveis de hemoglobina abaixo de 11 $\mathrm{g} / \mathrm{dL}$, somente $9,8 \%$ destas apresentavam concomitantemente ferro sérico abaixo de $50 \mu \mathrm{g} / \mathrm{dL}$ (Tabela 2). Foi encontrada uma diferença significativa ( $\mathrm{p}<0,05$, ANOVA) na concentração de hemoglobina entre os três trimestres, sendo o segundo semestre o que apresentou valor mais baixo (Figura 1). Quando avaliado a associação entre os níveis de hemoglobina com os de ferritina, foi observado que náo houve associação entre anemia e reserva de ferro corporal (Tabela 3).

Em relação ao estudo do ferro, coincidindo com os índices de anemia, 27,2\% das grávidas apresentaram níveis de ferro sérico abaixo dos valores de referência, $4 \%$ acima dos níveis considerados normais e $70 \%$ encontravam-se dentro da

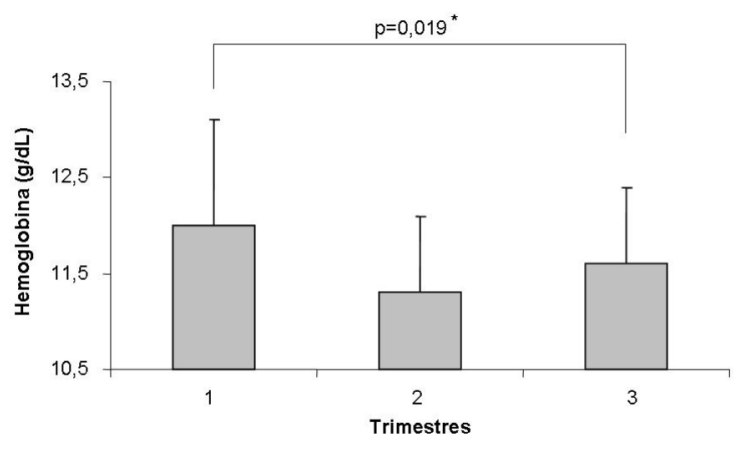

Figura 1 - Níveis de hemoglobina, portrimestre de gestação em mulheres atendidas na rede pública municipal de saúde, Manaus/AM, de agosto a outubro de 2006. * Diferença estatística entre os grupos (ANOVA)

Tabela 2 - Associação entre anemia e a deficiência de ferro em grávidas atendidas na rede pública municipal de saúde, Manaus/AM, de agosto a outubro de 2006.

\begin{tabular}{lccc}
\hline Hemoglobina & $\begin{array}{c}\text { Ferro }<50 \mu \mathrm{g} / \\
\mathrm{dL}(\%)\end{array}$ & $\begin{array}{c}\text { Ferro } \geq 50 \mu \mathrm{g} / \\
\mathrm{dL}(\%)\end{array}$ & Total (\%) \\
\hline$<11 \mathrm{~g} / \mathrm{dL}$ & 9,8 & 16,3 & 26,1 \\
$\geq 11 \mathrm{~g} / \mathrm{dL}$ & 17,4 & 56,5 & 73,9 \\
Total & 27,2 & 72,8 & 100 \\
\hline
\end{tabular}

$\mathrm{p}=0,208$, Odds Ratio $=2,096$ (IC $95 \%=0,80$ a 5,46 ) Teste do qui-quadrado.

Tabela 1 - Níveis médios de hemoglobina, hematócrito e marcadores do metabolismo do ferro em grávidas atendidas na rede pública municipal de saúde, Manaus/AM, de agosto a outubro de 2006.

\begin{tabular}{lccc}
\hline Parâmetro & $\begin{array}{c}\text { Não-anêmicas }(\mathrm{n}=68) \\
\mathrm{Hb} \geq 11 \mathrm{~g} / \mathrm{dL}\end{array}$ & $\begin{array}{c}\text { Anêmicas }(\mathrm{n}=24) \\
\mathrm{Hb}<11 \mathrm{~g} / \mathrm{dL}\end{array}$ & $\begin{array}{c}\rho \text {-valor* } \\
\text { Idade (anos) }\end{array}$ \\
$23 \pm 6$ & $22 \pm 6$ & 0,316 \\
Idade gestacional (semanas) & $19 \pm 9$ & $18 \pm 7$ & 0,757 \\
Hematócrito $(\mathrm{g} / \mathrm{dL})$ & $36,2 \pm 2,0$ & $32,1 \pm 1,2$ & $<0,001$ \\
Ferro $(\mu \mathrm{g} / \mathrm{dL})$ & $75 \pm 37$ & $55 \pm 24$ & 0,012 \\
CTLFe $(\mu \mathrm{g} / \mathrm{dL})$ & $387 \pm 73$ & $372 \pm 87$ & 0,411 \\
IST $(\%)$ & $21 \pm 12$ & $16 \pm 8$ & 0,144 \\
Transferrina $(\mathrm{mg} / \mathrm{dL})$ & $271 \pm 51$ & $260 \pm 61$ & 0,412 \\
Ferritina $(\mathrm{ng} / \mathrm{mL})$ & $28,8 \pm 22,9$ & $31,2 \pm 20,8$ & 0,671 \\
CLLFe $(\mu \mathrm{g} / \mathrm{dL})$ & $311 \pm 88$ & $317 \pm 97$ & 0,788 \\
\hline
\end{tabular}

CTLFe = Capacidade total de ligação do ferro; IST = Índice de saturação do ferro; CLLFe = Capacidade latente de ligação do ferro - *Teste $t$ de student 
Tabela 3 - Associação entre anemia e as reservas de ferro corporal em grávidas atendidas na rede pública municipal de saúde, Manaus/AM, de agosto a outubro de 2006.

\begin{tabular}{lccc}
\hline Hemoglobina & $\begin{array}{c}\text { Ferritina }<12 \mathrm{ng} / \\
\mathrm{dL}(\%)\end{array}$ & $\begin{array}{c}\text { Ferritina } \geq 12 \mathrm{ng} / \\
\mathrm{dL}(\%)\end{array}$ & Total $(\%)$ \\
\hline$<11 \mathrm{~g} / \mathrm{dL}$ & $0^{*}$ & 16,3 & 16,3 \\
$\geq 11 \mathrm{~g} / \mathrm{dL}$ & 20,9 & 62,8 & 83,7 \\
Total & 20,9 & 79,1 & 100
\end{tabular}

$\mathrm{P}=0,05$, Odds Ratio $=0,089$ (IC $95 \%=0,05$ a 1,55) Teste do qui-quadrado.

faixa de normalidade. Analisando-se o índice de saturaçâo da transferrina, encontramos $63 \%$ de IST inferior a $20 \%$. Por meio da dosagem de ferritina, observou-se que 20,9\% das gestantes incluídas no estudo possuíam níveis abaixo de $12 \mathrm{ng} / \mathrm{dL}$. O menor valor encontrado foi $9,8 \mathrm{ng} / \mathrm{dL}$. A ferritina também apresentou diferença significativa entre os trimestres ( $p<0,05$, ANOVA, sendo o terceiro trimestre o que apresentou a menor concentração média (Figura 2).

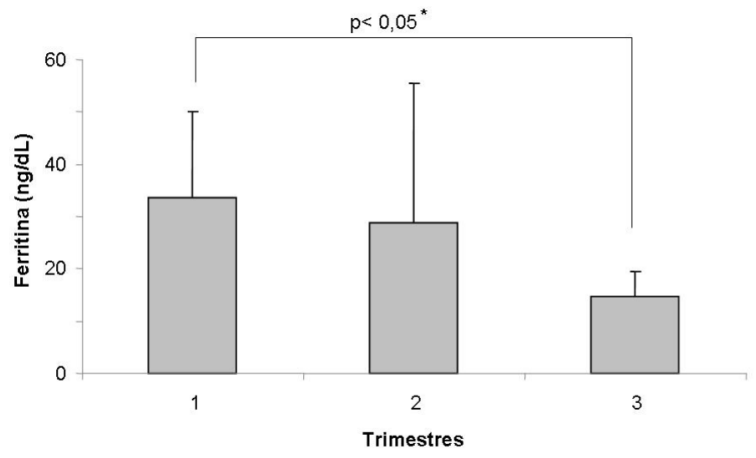

Figura 2 - Níveis de ferritina por trimestre de gestação em grávidas atendidas na rede pública municipal de saúde, Manaus/AM, de agosto a outubro de 2006. * Diferença estatística entre os grupos (ANOVA)

Comparando os resultados do presente trabalho com os de outros estudos, Szarfarc et al. (1985) encontraram 35,1\% de pacientes anêmicas; Van den Broek (1999) encontrou 58,1\%; Fujimori et al. (2000) encontraram 13,9\%; Méier et al. (2003) encontraram 31\%; Papa et al. (2003) encontraram 21,4\% e Idowu (2005), encontrou 76,5\%. Glover-Amengor et al. (2005) encontrou $57,1 \%$ de grávidas anêmicas, porém utilizou como ponto de corte para hemoglobina a concentração de $10 \mathrm{~g} / \mathrm{dL}$.

A concentraçáo de hemoglobina reflete uma situação de deficiência nutricional já estabelecida. A hemoglobina pode ser facilmente quantificada no sangue, e por isso, é o índice bioquímico adotado preferencialmente no diagnóstico da anemia (Szarfarc et al., 1982). Porém, tal exame deve ser analisado com cuidado em grávidas, pois as modificaçôes fisiológicas na composição sanguínea durante a gravidez, como a hemodiluição, dificultam o reconhecimento da condiçẫo patológica (Rezende, 2005). Na gravidez estáo agravadas as necessidades de ferro, sendo freqüente a anemia por carência desse elemento. Em virtude da hemodiluição, o nível mínimo de hemoglobina aceito é de $11 \mathrm{~g} / \mathrm{dL}$ e o de hematócrito, de 33\% (Fujimori et al., 2000; Rezende, 2005).

A concentração de hemoglobina variou nos três trimestres estudados, com valor mais baixo no segundo semestre. Fujimori et al. (2000) observou-se uma diminuiçáo de todos os parâmetros analisados no segundo semestre, mas somente a hemoglobina teve diferença significativa. Como já foi mencionado anteriormente, a hemodiluição característica da gravidez é um fator que dificulta o diagnóstico correto da anemia ou deficiência de ferro, sendo por esse motivo que Szarfarc et al. (1985) sugere que se refira o valor da concentraçấo de hemoglobina conjuntamente com a idade gestacional, uma vez que a hemodiluiçáo é mais acentuada no segundo semestre da gestação.

Com relação ao ferro sérico, onde no presente trabalho foram encontradas $27,2 \%$ de grávidas com níveis desse elemento abaixo do valor de referência, Guerra et al (1992) encontrou $10 \%$ de grávidas com níveis séricos de ferro baixos. Papa et al. (2003), estudando grávidas adolescentes, encontrou somente $4 \%$ de grávidas com níveis séricos de ferro abaixo do normal. Este é um parâmetro bastante utilizado, porém é pouco estável. Sua concentraçáo pode estar alterada na presença de processos infecciosos, diminuindo em poucas horas após o início da infecção. Também, a precisão do índice de saturação da transferrina é limitada, pois depende das concentraçốes de ferro e da capacidade latente de ligaçáo do ferro. Nas gestantes o IST encontra-se reduzido uma vez que o ferro sério encontra-se reduzido e o CLLF encontra-se aumentado (Paiva et al., 2000).

A ferritina é considerada um dos melhores marcadores para a avaliaçáo do estado de ferro corporal, pois analisa a quantidade estocada desse mineral. Porém esse teste também possui desvantagens, pois a ferritina pode aparecer como uma proteína de fase aguda, podendo estar aumentada devido a processo infeccioso ou inflamatório náo identificado (Papa et al.,2003). No presente trabalho foram encontradas $20,9 \%$ de grávidas com níveis de ferritina sérica abaixo dos valores recomendados. Contudo, este resultado pode ter sido mascarado, uma vez que aqui não foram avaliados os níveis séricos de proteína $\mathrm{C}$ reativa.

Os resultados apresentados neste trabalho devem ser vistos com atenção pelos profissionais de saúde que prestam assistências às grávidas para que estes possam dar as devidas orientaçôes acerca da importância da prevenção e do tratamento da anemia. Alguns autores defendem a teoria de que se deva fazer uma abordagem preventiva para o controle da deficiência de ferro e anemia gravídica, dirigida para adolescentes e mulheres em idade reprodutiva, com o objetivo de aumentar as reservas orgânicas do mineral nessa populaçáo (Fujimori et al., 2000). 
No presente trabalho foi observado que mais de um quarto das grávidas estudadas eram anêmicas pelo índice de hemoglobina e praticamente o mesmo número apresentava concentração de ferro sérico baixa. Isto fortemente sugere que se leve em consideração as características próprias de cada populaçáo na hora da escolha do melhor método a ser adotado na avaliação do metabolismo do ferro, como composição de ferro nos alimentos da região, índices hematológicos no início da gestação e presença de doenças infecciosas, inflamatórias ou hematológicas. Não há duvidas de que há uma redução nos níveis de hemoglobina e um aumento das necessidades de ferro na gestação, mas há também um aumento correlato da absorção do ferro e o ponto de equilíbrio está em saber até onde a queda da hemoglobina, que é fisiológica e recuperada no pós-parto.

\section{AGRADECIMENTOS}

À Secretaria de Saúde do Município de Manaus-AM, ao Laboratório Multicenter, à Fundaçáo de Amparo a Pesquisa do Estado do Amazonas (FAPEAM) pela Bolsa concedida a C.M.C e ao Conselho Nacional de Desenvolvimento Científico e Tecnológico (CNPq), pelo financiamento do projeto (Edital CT-SAÚDE/MCT/MS/CNPq 030/2004).

\section{BIBLIOGRAFIA CITADA}

ABESO - Associação Brasileira para o Estudo da Obesidade e da Síndrome Metabólica. Disponível em: <http://www.abeso.org. br>. Acesso em: 01/07/2007.

Brasil. Ministério da Saúde, Secretaria de Atenção à Saúde, Departamento de Atenção Básica. 2003. Política nacional de alimentação e nutrição. 2. ed. rev. - Brasília: Ministério da Saúde, 346pp.

Fujimori, E.; Laurenti, D.; Núñez de Cassana, L.M.; Oliveira, I.M.V.; Szarfarc, S.C. 2000. Anemia e deficiência de ferro em gestantes adolescentes. Revista de Nutrição, 13(3):177-184.

Glover-Amengor, M.; Owusu, W.B.; Akanmori, B.D. 2005. Determinants of anaemia in pregnancy in Sekyere west district, Ghana. Ghana Medical Journal, 39(3):102-107.

Guerra, E.M.; Barretto, O.C.O.; Pinto, A.V.; Castellao, K.G. 1992. Prevalência de deficiência de ferro em gestantes de primeira consulta em centros de saúde de área metropolitana, Brasil. Etiologia da anemia. Revista de Saúde Pública, 26(2):88-95.

Idowu, O.A.; Mafiana, C.F.; Sotiloye, D. 2005. Anaemia in pregnancy: a survey of pregnant women in Abeokuta, Nigeria. African Health Sciences, 5(4):295-299.
Meier, P.R; Nickerson, H.J.; Olson, K.A.; Berg, R.L.; Meyer, J.A. 2003. Prevention of iron deficiency anemia in adolescent and adult pregnancies. Clinical Medical Research, 1(1):29-36.

Paiva, A.A.; Rondo, P.H.C.; Guerra-Shinohara, E.M. 2000. Parâmetros para a avaliação do estado nutricional de ferro. Revista de Saúde Pública, 34(4):421-426.

Papa, A.C.E.; Furlan, J.P.; Pasquelle, M.; Guazzelli, C.A.F.; Figueiredo, M.S.; Camano, L.; Mattar, L. 2003. A Anemia por deficiência de ferro na grávida adolescente - Comparação entre Métodos Laboratoriais. Revista Brasileira de Ginecologia e Obstetricia, 25(10):731-738.

Resende, J. 2005. Obstetrícia. 10. ed. Guanabara Koogan: Rio de Janeiro. 1565pp.

Souza, A.I.; Batista-Fiho, M. 2003. Diagnóstico e tratamento das anemias carências na gestação: consensos e controvérsias. Revista Brasileira de Saúde Materno Infantil, 3(4):473-479.

Souza, A.I.; Batista-Filho, M.; Ferreira, L.O.C. 2002. Alterações hematológicas da gravidez. Revista Brasileira de Hematologia e Hemoterapia, 24(1):29-36.

Szarfarc, F.C. 1985. A anemia nutricional entre gestantes atendidas em centros de saúde do Estado de São Paulo (Brasil). Revista de Saúde Pública, 19:450-457.

Szarfarc, S.C.; Siqueira, A.A.F.; Martins, I.S.; Tanaka, A.C.D. 1982. Estudo comparativo de indicadores bioquímicos de concentração de ferro, em duas populaçôes de gestantes, com e sem atendimento pré-natal. Revista de Saúde Pública, 16:1-6.

Van den Broek, N.R.; Ntonya, C.; Mhango, E.; White, S.A. 1999. Diagnosing anaemia in pregnancy in rural clinics: assessing the potential of the Hemoglobin Colour Scale. Bulletin World Health Organization, 77(1):15-21.

Verrastro, T. 2002. Hematologia e hemoterapia: fundamentos de morfologia, fisiologia, patologia e clínica. Atheneu: São Paulo. 303pp.

World Health Organization. 2000. Making pregnancy safer - a health sector strategy for reducing maternal and e perinatal morbidity and mortality. Geneva: World Health Organization. 25pp.

World Health Organization. 2004. Focusing on anemia. Geneva. Disponível em: <http://www.paho.org/English/AD/FCH/NU/ WHO04_Anemia.pdf> Acesso 01/07/2007.

Recebido em 25/01/2008

Aceito em 22/09/2009 
